\title{
Keynote: Primitives for Physical Trust
}

\author{
Anish Arora \\ Department of Computer Science and Engineering \\ The Ohio State University \\ Columbus Ohio 43210, USA \\ anish@cse.ohio-state.edu
}

\begin{abstract}
This talk explores the question of whether wireless communication can achieve security - confidentiality (unicast and broadcast), authentication, non-repudiation, anonymity - without assuming any shared secrets and using only low-cost computation primitives. The key idea is to exploit physical characteristics of the network medium to develop a basis of physical primitives that suffice for rethinking a security protocol suite.
\end{abstract}

